\title{
Statins for primary prevention: identifying low-risk individuals
}

Yu-li Huang, Yi Huang and Yan-xian Wu

We read the Review by Željko Reiner (Statins in the primary prevention of cardiovascular disease. Nat. Rev. Cardiol. doi:10.1038/ nrcardio.2013.80) $)^{1}$ with great interest. We are particularly interested in the role of statins in primary prevention of cardiovascular disease (CVD) in individuals at low cardiovascular risk.

As was well discussed in the Review, clear evidence exists to support the use of statins for primary prevention in high-risk individuals. ${ }^{1}$ A risk of developing CVD $>20 \%$ over 10 years, or $>10 \%$ over 5 years, is widely used as the cut-off point for prescribing statins. ${ }^{2}$ However, the use of statins in lowrisk individuals is controversial. A metaanalysis from the Cholesterol Treatment Trialists' (CTT) Collaborators showed that statins reduce the risk of major cardiovascular events by about $20 \%$ across all levels of baseline risk (even in individuals with a 5 -year risk of major vascular events that is $<10 \%){ }^{3}$ The CTT Collaborators suggested that the current guidelines for CVD prevention might need to be reconsidered. ${ }^{3}$ Their study raises enthusiasm for statins in the primary prevention of CVD in individuals at low cardiovascular risk. In fact, some have advocated treating 'all comers' with statins. ${ }^{4}$ Several issues, however, should be considered before advocating the widespread use of statins in individuals at low risk, such as the feasibility, desirability, and cost-effectiveness of such a strategy, and the quality of life for apparently healthy individuals who are prescribed lifelong drug therapy. Also important are potential adverse effects of statins. $2,5,6$

We agree that statins are likely to be beneficial to patients with low cardiovascular risk calculated using traditional risk-assessment algorithms, such as the Framingham Risk Score and the SCORE (Systematic COronary Risk Estimation) charts. ${ }^{4}$ However, additional risk-stratification options are needed to guide the use of statins in low-risk individuals. Data from the JUPITER trial ${ }^{7}$ showed that individuals with an elevated level of C-reactive protein measured by the highsensitivity assay (hs-CRP) benefit from statin use, regardless of their LDL-cholesterol level. After this trial, hs-CRP is becoming accepted as a novel cardiovascular risk factor. ${ }^{7}$

Coronary artery calcium (CAC) scoring has been shown to predict coronary events beyond the use of the traditional Framingham Risk Score, with a high CAC burden $(\geq 300)$ being associated with increased risk. ${ }^{8,9}$ Data from comparative effectiveness studies have suggested that quantification of CAC is a superior method to measurement of the hs-CRP level for improving risk assessment. ${ }^{10}$ Investigators in the St. Francis Heart Study ${ }^{11}$ enrolled patients with an elevated CAC level (CAC $>80^{\text {th }}$ percentile) and randomly allocated them to receive $20 \mathrm{mg}$ of atorvastatin daily or placebo. The overall result did not meet statistical significance $(P=0.08)$ because of the limited power of the trial, but showed that atorvastatin significantly reduced the composite rate of adverse events from coronary heart disease by $30 \%{ }^{11}$

In conclusion, the use of statins for primary prevention in low-risk individuals remains an ambiguous area. We believe that additional risk-stratification options (such as measurement of the hs-CRP level and CAC scoring) should be explored in this population. A new risk-assessment algorithm that includes the traditional risk measures, but also incorporates these novel markers should be developed to identify low-risk patients and guide the prophylactic use of statins.

\section{Department of Cardiology, the Affiliated} Hospital at Shunde (the First People's Hospital of Shunde), Southern Medical University, Penglai Road 1, Daliang Town, Shunde District, Foshan 528300, People's Republic of China (Y.-I. Huang, Y.-x. Wu). The Second Clinical Medicine Institute, Guangdong Medical College, Dongguan, People's Republic of China (Y. Huang).

Correspondence to: Y.-I. Huang

hyuli821@163.com
Acknowledgements

The authors of this manuscript are funded by the Medical Scientific Research Grant of the Health Ministry of Guangdong province, China

(No. B2011310, No. A2012663), and Scientific Research Fund of Foshan, Guangdong, China (No. 201208210).

\section{Competing interests}

The authors declare no competing interests.

1. Reiner, Z. Statins in the primary prevention of cardiovascular disease. Nat. Rev. Cardiol. http://dx.doi.org/10.1038/nrcardio.2013.80.

2. Smeeth, L. \& Hemingway, H. Improving vascular health: are pills the answer? BMJ 344, e3802 (2012).

3. Mihaylova, B. et al. The effects of lowering LDL cholesterol with statin therapy in people at low risk of vascular disease: meta-analysis of individual data from 27 randomised trials. Lancet 380, 581-590 (2012).

4. Madanieh, R., Hasan, R. K., Anusionwu, O. F., Blumenthal, R. S. \& Blaha, M. J. Cardiovascular disease prevention: matching evidence-based algorithms with individualized care. Clin. Pharmacol. Ther. 93, 321-323 (2013).

5. Ebrahim, S. \& Casas, J. P. Statins for all by the age of 50 years? Lancet $\mathbf{3 8 0}, 545-547$ (2012).

6. Taylor, F. et al. Statins for the primary prevention of cardiovascular disease. Cochrane Database of Systematic Reviews Issue 1. Art. No.: CD004816. http://dx.doi.org/10.1002/ 14651858.CD004816.pub4.

7. Ridker, P. M. et al. Rosuvastatin to prevent vascular events in men and women with elevated C-reactive protein. N. Engl. J. Med. 359, 2195-2207 (2008).

8. Lakoski, S. G. et al. Coronary artery calcium scores and risk for cardiovascular events in women classified as "low risk" based on Framingham risk score: the multi-ethnic study of atherosclerosis (MESA). Arch. Intern. Med. 167, 2437-2442 (2007).

9. Kondos, G. T. et al. Electron-beam tomography coronary artery calcium and cardiac events: a 37-month follow-up of 5635 initially asymptomatic low- to intermediate-risk adults. Circulation 107, 2571-2576 (2003).

10. Blaha, M. J. et al. Associations between C-reactive protein, coronary artery calcium, and cardiovascular events: implications for the JUPITER population from MESA, a populationbased cohort study. Lancet 378, 684-692 (2011).

11. Arad, Y., Spadaro, L. A., Roth, M., Newstein, D. \& Guerci, A. D. Treatment of asymptomatic adults with elevated coronary calcium scores with atorvastatin, vitamin $\mathrm{C}$, and vitamin $\mathrm{E}$ : the St. Francis Heart Study randomized clinical trial. J. Am. Coll. Cardiol. 46, 166-172 (2005). 